\title{
Mathematical model development for superplastic forming of sheet shells by subliming agent gas pressure
}

\author{
Oleksandr Anishchenko ${ }^{1, *}$, Volodymyr Kukhar ${ }^{1}$, Viktor Agarkov ${ }^{2}$, and Alla Vorobeva $^{3}$ \\ ${ }^{1}$ Metalforming department, Pryazovskyi State Technical University, Mariupol, Ukraine \\ ${ }^{2}$ State Enterprise "Kharkiv Regional Research and Production Center for Standardization, Metrology \\ and Certification", Kharkiv, Ukraine \\ ${ }^{3}$ Moscow state University of technology and management. K.G. Razumovsky, Zemlyanoy Val street, \\ 73, 109004, Moscow, Russia
}

\begin{abstract}
The authors presented in the article an analytical model of superplastic forming of spherical shells by pressure of the gas (gaseous phase) created upon sublimation of sublimate agent. Sublimate was placed in a hermetic cavity between the workpiece and the presser cover and heated to the temperature of its sublimation. The model uses the Berthelot equation for the state of real gases, the Bernoulli relation, and the F. Jovane equations for adjusting the shell forming pressure as a function of its relative height, the heat balance equation for the sublimation process, and the Clausius-Clapeyron relation for sublimation of substances. The authors showed that the superplastic forming pressure control can be effectively applied by the way of change in the forming temperature relative to temperature of sublimate agent sublimation. The experimental results of forming of shells from aluminum alloys AlMg3Mn, AlMg5Mn and AlMg6Mn, confirming theoretical calculations of the velocity and temperature conditions of superplastic forming by the sublimating agent pressure.
\end{abstract}

\section{Introduction}

Superplastic forming of spherical shells from sheets is performed by a gas medium, and aluminum and titanium based alloys are preferably formed [1-5]. The application of a gas medium (inert gases mainly [6-8]) is associated with the use of compressor plants and pipelines that are hermetically sealed into forming dies [8,9]. An important advantage of superplastic forming is not the use of press equipment and the elimination of a number of problems in the area of ensuring the accuracy of the "press-die" system and occupation safety [10-14]. Despite the study of the processes of gas superplastic forming $[1,3,7,8$, 15] and the progress in the methods of material heating before forming [16, 17], the technical realization of multistage molding operations, especially with respect to the

\footnotetext{
*Corresponding author: alexander.anishchenko@gmail.com
} 
required load (force) regimes, remains very complicated [7, 8, 18-20]. Replacement of the external gaseous medium to the internal gas medium (that created directly in the die cavity) will significantly facilitate the technological realization of the gas superplastic forming (Fig. 1), while it is obvious that the methods for taking into account the material plastic properties and the designing of the passes will remain the same [5, 6, 8, 15, 21-23]. Difficulties are expected at the stage of calculation of load (force) behavior, depending on the method of creating internal die cavity gas pressure.
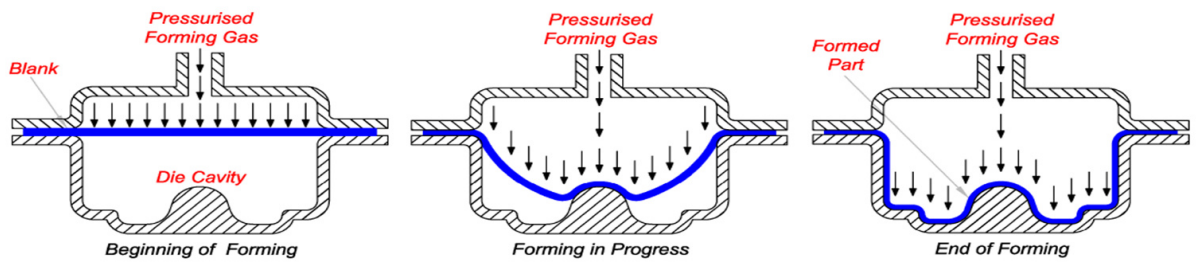

Fig. 1. Stages of gas superplastic forming [22].

One of the modern and progressive variants of superplastic forming is the molding of shells by the pressure of a gaseous medium formed as a result of a change in the aggregate state of thermo-metastable substance (sublimate or destruct agent) in the hermetic die cavity $[8,9]$. Based on this kind of gas forming, in this work an attempt is made to determine the optimal power regime in relation to free forming of dome-shaped shells due to the controlled sublimation in the die cavity. Preliminary analysis has shown that a sufficiently large number of factors (mass, density, surface area, critical temperature of the sublimate, geometric dimensions of the preform, die, forming temperature, etc.) affecting the formation of domes cause significant difficulties in the experimental determination of optimal gas-forming regimes with controlled sublimation of subliming agent. Therefore, the problem was solved theoretically and tested experimentally with the example of free bulging of workpiece from Magnalium with a radius of $r=46 \mathrm{~mm}$ in a die with a clamping lid with a diameter of $D=92 \mathrm{~mm}$ and a height of $135 \mathrm{~mm}$.

\section{Methodology}

Calculation of superplastic forming is based on the theory of momentless shells [24-26]. The following assumptions are made: deformation is triaxial and axisymmetric; the stress state is flat and axisymmetric, the incompressibility conditions, deformation hardening, elastic deformations, the anisotropy of the mechanical properties of the molded material and dynamic loads are negligibly small. In addition, it was assumed that the thinning of the shell walls is uniform, and the temperature of the die surface on contact with the sublimate layer remains constant and equal to the forming temperature. It was assumed that the heat transfer in the cavity of the die is carried out by radiation, and convection and thermal conductivity can be neglected, due to the absence of communication with the external medium and the formation, upon sublimation, of the gas cushion separating the die walls and the surface of the sublimate in the solid state.

As a "reference" law for regulating gas pressure during forming into a cylindrical cavity, the Jovane ratio [27] was chosen:

$$
p / p_{0}=\left[H\left(1+H_{0}^{2}\right)^{2}\right] /\left[H_{0}\left(1+H^{2}\right)^{2}\right],
$$


where $H$ - relative height of the shell, $H=h / r$ ( $h$ is the height of the shell); $H_{0}-$ initial relative height of the shell; $p$ - forming pressure; $p_{0}$ - initial forming pressure corresponding to the height $H_{0}$.

The parameters of the formation of $H_{0}$ and $p_{0}$ were determined from the known dependences of the mechanics of continuous media [3] and on the basis of the equation for the elastically viscous plastic medium [1]. The increase in forming pressure during sublimation in the die cavity to a maximum, i.e., until the temperature of the sublimation reaches the forming temperature, is described by the combination of the following dependencies:

- the equation of state of a mixture of real gases developed by Berthelot:

$$
\left(d m / \mu_{m}\right) R T=(V+b) d p+(p+c) d V ;
$$

- the chemical equation of the sublimation reaction of sublimate agent arbitrary mass:

$$
\mu_{M} f_{M} d m=\mu_{m} \sum_{i=1}^{N} f_{m_{i}} d M
$$

- the equation of the thermal balance of the sublimation process:

$$
F \sigma E\left(T^{4}-T_{M}^{4}\right) d t=q d M
$$

- the Clausius-Clapeyron equation for the sublimation of substances:

$$
R T_{M}^{2} d p=q p d T_{M}
$$

- the ratio that determines the volume of the gaseous phase in the closed die cavity:

$$
V=\pi D^{2} l / 4-M / \rho+\pi \cdot a^{3} H\left(H^{2}+3\right) / 6 ;
$$

- the equation for calculating the duration of forming at a given optimal strain rate:

$$
\xi\left(1+H^{2}\right) d t=2 H d H
$$

- ratio that relating the surface area changing and sublimate agent mass with the thickness of its layer:

$$
\begin{gathered}
d F=4 \pi(\alpha+1) S_{M} d S_{M} \\
S_{M}=\sqrt[3]{M / \pi \alpha^{2} \rho}
\end{gathered}
$$

of which

$$
d F=\left[4 \pi(\alpha+1) / 3 \sqrt[3]{\pi^{2} \alpha M \rho^{2}}\right] d M .
$$

In the dependences (2) - (10): $M$ - mass of the sublimate agent; $m$ - mass of the gaseous phase; $\mu_{M}, \mu_{m}$ - accordingly molar masses of the sublimate and mixtures of 
gases created during its sublimation; $R$ - universal gas constant; $T$ - forming temperature; $K$ - volume of the gaseous phase; $b, c$-correction factors in the Berthelot equation; $f_{m}, f_{M}$ - stoichiometric coefficients of the sublimation reaction; $T_{M}$ - critical temperature of the sublimate agent; $F$ - surface area of the sublimate agent; $\sigma$ - StefanBoltzmann constant; $E$ - reduced degree of blackness of bodies surface; $q$ - latent heat of sublimation; $t$ - forming time; $l$ - cavity depth of the presser cap of the die; $\rho$ - density of the sublimate agent; $\alpha=a / S_{M}, a, S_{M}$ - radius and thickness of the sublimate layer in the die cavity, respectively.

The maximum correspondence of the pressure increase at sublimation of sublimate agent with the ascending branch of the "reference" dependence (1) at the section $H_{0} \leq H \leq 1 / \sqrt{3}$ was established by means of a computer calculation by varying within the specified limits of parameters entering into equations (2)-(10). At the stage of shell forming with height $H=1 / \sqrt{3}$ the necessary pressure decrease was determined by formula (5). At the same time, the forming temperature was reduced by shutting down the die heating system, which caused the interfacial equilibrium of the sublimate to equal at forming and sublimation temperatures.

For the experimental verification of the proposed model of gas superplastic forming at all its stages and taking into account the fact that the velocity and temperature ranges of the superplastic deformation of the Magnaliums, $\xi=10^{2} \ldots 10^{-4} \mathrm{~s}^{-1}, T=380 \ldots 450^{\circ} \mathrm{C}$, in theoretical calculations the forming temperature was assumed as $450{ }^{\circ} \mathrm{C}$ in order to increase the possible range of its change when forming domes in height $H \geq 1 / \sqrt{3}$, and the strain rate varied only in the above-mentioned limits in increments of $\Delta \xi=2 \cdot 10^{-4} \mathrm{~s}^{-1}$.

\section{Results, verification and discussion}

The optimum values of the parameters of the gasostatic forming calculated with a computer with a minimum gradient of the strain rate were verificated by forming to crashing the walls of the domes from alloys $A l M g 3 M n, A l M g 5 M n$, and $A l M g 6 M n$ with initial thickness of workpieces $S_{0}=0.6 \ldots 1.0 \mathrm{~mm}$ (when using the sublimate $\mathrm{NH}_{4} \mathrm{Cl}$ ).

Optimum parameters of the superplastic forming see Table 1.

Table 1.Optimum parameters of the superplastic forming.

\begin{tabular}{|c|c|c|c|c|c|}
\hline Alloy & $M, \mathrm{~kg}$ & $\xi, \mathrm{s}^{-1}$ & $\alpha$ & $V_{m}, \mathrm{~cm}^{3}$ & $S_{0}, \mathrm{~mm}$ \\
\hline$A l M g 5 M n$ & 0.9 & $8 \times 10^{-4}$ & 1.3 & 30 & 1 \\
\hline$A l M g 6 M n$ & 0.75 & $6 \times 10^{-4}$ & 2.25 & 25 & 0.6 \\
\hline$A l M g 3 M n$ & 0.75 & $6 \times 10^{-4}$ & 2.25 & 25 & 0.6 \\
\hline
\end{tabular}

For comparison; chose the ratio of the calculated and experimentally determined by the equation (7) deformation rates of shell. Under the experimental conditions (Fig. 2), the error of calculation on a computer that smaller than higher the level of superplastic properties of the workpiece material and the greater the geometric dimensions of the workpiece. So, ratio of the theoretical and experimental data for workpiece formed of AlMg5Mn alloy with thickness $S_{0}=1.0 \mathrm{~mm}$ is 2.46 . With a decrease in the relative 
thickness $S_{0} / r$ of the workpiece the error is reduced to 1.88 (AlMg6Mn alloy) and 0.94 ( $A l M g 3 M n$ alloy). With the same geometric dimensions of the workpieces, the index of the unevenness of the strain rate for the magnesium $A l M g 3 M n$ is lower than for $A l M g 6 M n$.

Despite the high error in the theoretical calculation, the results obtained can be considered satisfactory, since in all experiments the absolute values of the strain rate for shells did not exceed the limits of superplasticity values and amounted: to alloy $A l M g 5 \mathrm{Mn}$ is $\xi=2.16 \cdot 10^{-4} \ldots 2.18 \cdot 10^{3} \mathrm{~s}^{-1}$; to alloy AlMg6Mn $\xi=1.3 \cdot 10^{-4} \ldots 1.3 \cdot 10^{-2} \mathrm{~s}^{-1}$; to alloy AlMg3Mn $\xi=5.76 \cdot 10^{-3} \ldots 1.33 \cdot 10^{-2} \mathrm{~s}^{-1}$.

The decrease in temperature, determined theoretically, as well as its change in the course of experiment, show that at the instant of shell rupture $(H \leq 1.1)$ is temperature $T \geq 420^{\circ} \mathrm{C}$, i.e. the forming temperature was in the optimum range of superplastic deformation for the investigated alloys.

Maximum height of formed shells of AlMg5Mn, AlMg6Mn and AlMg3Mn alloys was increased to $16.2,21$ и $24 \%$ respectively. Increasing the resource of the deformation ability of alloys without significantly complicating of forming technology, applied equipment and rigging testifies to the prospects of industrial use of the proposed mode of superplastic forming.

Despite the high error in the theoretical calculation, the results obtained can be considered satisfactory, since in all experiments the absolute values of the strain rate for shells did not exceed the limits of superplasticity values and amounted: to alloy $A l M g 5 M n$ is $\xi=2.16 \cdot 10^{-4} \ldots 2.18 \cdot 10^{3} \mathrm{~s}^{-1} ; \quad$ to alloy $A l M g 6 M n$ is $\xi=1.3 \cdot 10^{-4} \ldots 1.3 \cdot 10^{-2} \mathrm{~s}^{-1}$; to alloy $\mathrm{AlMg} 3 \mathrm{Mn}$ is $\xi=5.76 \cdot 10^{-3} \ldots 1.33 \cdot 10^{-2} \mathrm{~s}^{-1}$.

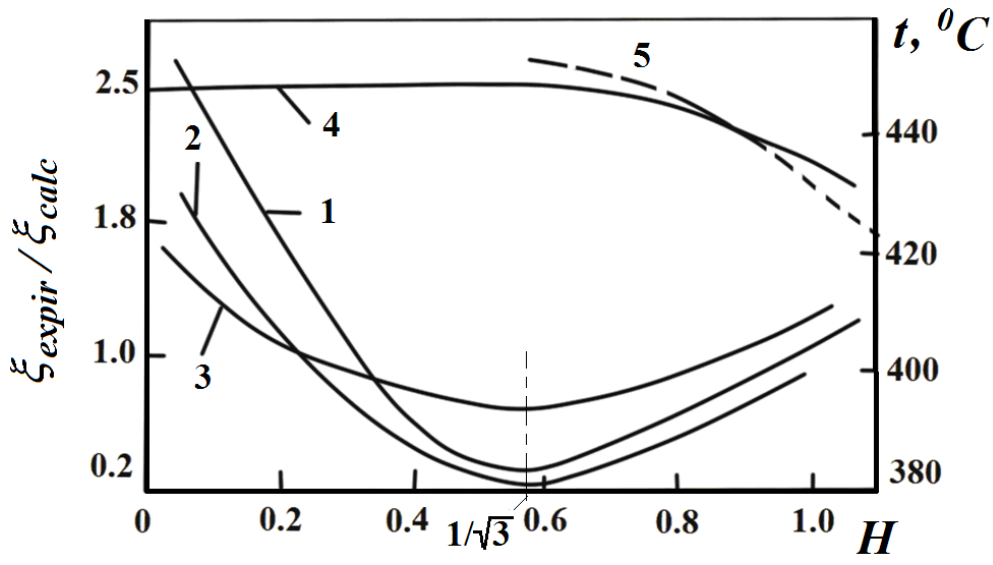

Fig. 2. Comparison of calculated and experimental values of strain rates $(1,2,3)$ and temperature change $(4,5)$ in process of domes forming, $\xi_{\text {exp }}$ ir,$\xi_{\text {calc }}$ - experimental and calculated strain rate: 1 - AlMg5Mn alloy; 2 - AlMg6Mn alloy; 3 - AlMg3Mn alloy; 4 - experimental values of forming temperature; 5 - lowering of temperature (calculated according to the Clapeyron-Clausius equation) at the stage of forming domes with height $H \geq 1 / \sqrt{3}$.

The decrease in temperature, determined theoretically, as well as its change in the course of experiment, show that at the instant of shell rupture $(H \leq 1.1)$ is temperature $T \geq 420{ }^{\circ} \mathrm{C}$, i.e. the forming temperature was in the optimum range of superplastic deformation for the investigated alloys.

Maximum height of formed shells of AlMg5Mn, AlMg6Mn and AlMg3Mn alloys was increased to $16.2,21$ и $24 \%$ respectively. Increasing the resource of the deformation ability of alloys without significantly complicating of forming technology, applied equipment and 
rigging testifies to the prospects of industrial use of the proposed mode of superplastic forming.

\section{Conclusions}

1.An analytical model of superplastic forming of spherical shells by the pressure of a gas medium created during the sublimation of sublimate agent in the die cavity is developed.

2.It is shown that the control of the pressure of the gaseous medium can be effectively performed by a corresponding change in the forming temperature relative to the sublimate agent sublimation temperature.

3.The correspondence of the velocity behavior of shell superplastic forming to theoretically calculated values according to the developed model was experimentally confirmed.

\section{References}

1. S.A. Aksenov, et al., Determination of optimal gas forming conditions from free bulging tests at constant pressure. J. of Mat. Proc. Tech. 217, 158-164, (2015) doi: 10.1016/j.jmatprotec.2014.11.015

2. S.A. Aksenov, et al., Characterization of Superplastic Materials by Results of Free Bulging Tests. Mat. Scie. Forum 838-839, 552-556 (2016) doi:10.4028/www.scientific.net/MSF.838-839.552

3. S.A. Aksenov, A.V. Kolesnikov, A.V. Mikhaylovskaya, Design of a gas forming technology using the material constants obtained by tensile and free bulging testing. J. of Mat. Proc. Tech. 237, 88-95 (2016) doi: 10.1016/j.jmatprotec.2016.06.003

4. M. Reza, A.N. Zuelli, Metrology and Microscopy Analysis of Multisheet Packs Manufactured via Superplastic Forming to Study Possible Diffusion Bonding. Proc. Eng. 183, 251-256 (2017) doi: 10.1016/j.proeng.2017.04.031

5. E.N. Chumachenko, Modeling of superplastic forming of titanium-alloy shells at reduced temperatures. Mech. of Solids 39(6), 116-128 (2004)

6. J. Bonet, et al., Simulating superplastic forming. Comp. Methods in Appl. Mech. and Eng. 195(48-49), 6580-6603 (2006) doi: 10.1016/j.cma.2005.03.012

7. S. Lee, J.S. Tang, C.L. Chu, Prior sheet buckling leading to wrinkling formation in a gas forming a V-trough with wavy bottom. J. of Manufact. Proc. 21, 101-106 (2016) doi: 10.1016/j.jmapro.2015.12.001

8. R. Chatterjee, J. Mukhopadhyay, A Review of Super plastic forming. Materials Today: Proceedings 5(2) part 1, $4452-4459$ (2018) doi: 10.1016/j.matpr.2017.12.014

9. Y. Luo, et al., Development of an advanced superplastic forming process utilizing a mechanical pre-forming operation. Intern. J. of Mach. Tools and Manufact. 48 (1213), 1509-1518 (2008) doi: 10.1016/j.ijmachtools.2007.12.010

10. J.C. Ng, et al., Validation of a modified material model for use with shell elements to improve the predictive accuracy of the thickness distribution in superplastic forming of sheet metals. J. of Materials Proc. Techn. 211, 1386-1394 (2011) doi: 10.1016/j.jmatprotec.2011.03.012

11. E. Balalayeva, et al., Researching of the Stress-Strain State of the Open-Type Press Frame Using of Elastic Compensator of Errors of "Press-Die" System. Adv. in Intell. Syst. and Comp. 692, 220-235, Springer (2018) doi: 10.1007/978-3-319-70987-1_24 
12. V. Kukhar, E. Balalayeva, O. Nesterov, Calculation method and simulation of work of the ring elastic compensator for sheet-forming. In: MATEC Web Conf. (ICMTMTE 2017) 129, pp. 01041 (2017) doi: 10.1051/matecconf/201712901041

13. Kukhar V. et al.: Estimation of Occupation Safety Risks at Energetic Sector of Iron and Steel Works. International Journal of Engineering \& Technology (UAE) 7(2.23), 216220 (2018). doi: 10.14419/ijet.v7i2.23.11922.

14. A.S. Anishchenko, N.Yu. Sosnovskij, Rolling Machines for Washing Machines Bodies Working. Kuznechno-Shtampovochnoe Proizvodstvo 11, 27-27 (1993)

15. G. Giuliano, Superplastic Forming of Advanced Metallic Materials: Method and Applications. Oxford-Cambridge-Philadelfia-New Dehli: Woodhead Publishing Ltd. (2011)

16. D. Mauduit, et al, Industrial applications of the superplastic forming by using InfraRed heater. Proc. Eng. 207, 1898-1903 (2017) doi: 10.1016/j.proeng.2017.10.958

17. V. Kukhar, et al., Designing of induction heaters for the edges of pre-rolled wide ultrafine sheets and strips correlated with the chilling end-effect. MEES'2017, pp. 404-407, Kremenchuk Mykhailo Ostrohradskyi National University, Kremenchuk, Ukraine (2017) doi: 10.1109/MEES.2017.8248945

18. G. Jr.Luckey, P. Friedman, K. Weinmann, Design and experimental validation of a two-stage superplastic forming die. J. of Mat. Proc. Tech. 209, 2152-2160 (2009) doi: 10.1016/j.proeng.2014.10.145

19. F. Jarrar, Designing gas pressure profiles for AA5083 superplastic forming. Proc. Eng. 81, 1084-1089 (2014) doi: 10.1016/j.proeng.2014.10.145

20. V. Kukhar, et al., Experimental Research and Method for Calculation of 'Upsettingwith-Buckling' Load at the Impression-Free (Dieless) Preforming of Workpiece. In: E3S Web of Conf. (HRC 2017) 33, pp.02031 (2018) doi: $10.1051 /$ e3sconf/20183302031

21. A.S. Anishchenko, Heat treatment effect on properties of deformed alloy type 36N. 2, 31-32 (1996)

22. F.K. Abu-Farha, M.K. Khraishen, An integrated approach to the Superplastic Forming of alloys: toward sustainable manufacturing. Int. J. Sustainable Manufact. 1, 1-2 (2008) doi: 10.1504/IJSM.2008.019225

23. F. Gao, et al., Rheological Law and Constitutive Model for Superplastic Deformation of Ti-6Al-4V. J. of Alloys and Compounds 701, 177-185 (2017) doi: 10.1016/j.jallcom.2017.01.096

24. I. Shatskyi, I. Popadyuk, A. Velychkovych, Modelling of energy dissipation in shell dampers. In: Fuis, V. (ed.) Book of full texts. Eng. Mech., $23^{\text {rd }}$ Int. Conf., Svratka, Czech Republic, pp. 870-873, 15-18 (2017)

25. R. Puzyr, et al., Development of a method to determine deformations in the manufacture of a vehicle wheel rim. East.-Eur. J.1 of Enterp. Tech. 4, 55-60 (2018) doi: 10.15587/1729-4061.2018.139534

26. R. Puzyr, et al., Experimental Study of the Process of Radial Rotation Profiling of Wheel Rims Resulting in Formation and Technological Flattening of the Corrugations. Manufact. Tech. 18(1), 106-111 (2018) doi: 10.21062/ujep/61.2018/a/1213$2489 / \mathrm{mt} / 18 / 1 / 106$

27. F. Jovane, An approximate analysis of the superplastic forming of a thin circular diaphragm: theory and experiments. Int. J. Mech. Scie. 10(6), 409-427 (1968) 